\title{
Zu den Klassenkämpfen in Chile
}

\author{
Interview mit Urs Muller-Plantenberg
}

Probleme des Klassenkampfs: Engels sagt, daß man um die Armee nicht herumkommt -. man könne sie nur mit gleich starken Kräften besiegen oder sie zersetzen. Es wird bei uns seit dem Putsch im September vielfach die Auffassung vertreten, Allende habe die Arbeiter verraten, indem er den Aufbau von Arbeitermilizen verhindert und sogar deren Entwaffnung zugestimmt habe. Auf der anderen Seite habe er die Armee gestützt und sogar gefördert (durch Waffenkäufe im Ausland usw.). Was würdest du zu dieser Auffassung sagen?

Müller-Plantenberg: Engels hat recht. Entweder mus man die Armee mit gleich starken oder möglichst stärkeren Mitteln besiegen oder sie zersetzen. Aber was ergibt sich daraus? Die Kräfte, die die Armee besiegen oder zersetzen könnten, müssen doch zuerst geschaffen sein. Vielfach wird behauptet, daß 1970 die Arbeiter bewußt und entschlossen genug gewesen wären, eine bewaffnete Revolution durchzuführen. Diese Behauptung geht von völlig falschen Voraussetzungen aus. Die Bewaffnung eines Arbeiters, der sich seiner Klassensituation nicht voll bewußt ist, der noch Illusionen über die tatsächliche Klassensituation hat, nuitzt gar nichts, weil er nicht weiß, was er mit der Waffe in der Hand anfangen soll. Im Laufe der drei Jahre der Regierungszeit Allendes hat ein Prozeß stattgefunden, der das Bewußtsein der Arbeitermassen unglaublich gefördert hat, der ihnen Klarheit über ihre Klassenlage gegeben hat, der zu einer Polarisierung der gesamten Bevölkerung geführt hat und in dem schließlich auch die Arbeiter erkannt haben, daß sie sich selbst bewaffnen müssen.

Nun wird gesagt, daß die Regierung Allende diese Bewaffnung verhindert habe und daß sie sogar die Arbeiter, die bereit waren, sich zu bewaffnen, entwaffnet habe. Jeder Versuch von seiten der Regierung, die Arbeiter ganz offiziell zu bewaff. nen, hätte sofort zu einem Blutbad geführt, wie wir es jetzt im September 1973 erleben, aber in einer Situation, in der die Arbeiter tatsächlich noch nicht die Klassenkampferfahrung gemacht hatten, die sie jetzt gewonnen haben. Die Bewaffnung der Arbeiter konnte also nur von ihnen selbst aus erfolgen bzw. von ihren Organisationen, d. h. den Parteien, Gewerkschaften usw. Aber die Parteien konnten, zumindest soweit sie in der Regierung waren, die Bewaffnung der Arbeiter auch nicht offiziell fördern oder ganz offiziell betreiben. Was sie tun konnten, war, die Bewaffnung im Geheimen durchzuführen und das allgemeine Bewußtsein von ihrẹ Notwendigkeit zu schaffen.

Daneben bestand die Aufgabe, Teile des Militärs auf die eigene Seite zu ziehen. Das ist nicht mit Systematik betrieben worden, sondern der Klassenkampf selbst hat vielen Soldaten und Matrosen allmählich klargemacht, daß ihre Interessen nicht mit denen der Generäle, sondern mit denen der Arbeiter identisch sind. Das Bewußtsein über diese Situation entstand vor allem durch den Angriff der Rechten 
auf die tatsächliche Lebenssituation sowohl des Arbeiters wie des Soldaten. Das wirtschaftliche Chaos, das von der Rechten, von der Bourgeoisie und den mit ihr kollaborierenden politischen Gruppen in den letzten Monaten bewußt gesteigert wurde, hat auch bei vielen Soldaten und Matrosen zu einer immer stärkeren Klarheit uber ihre Stellung in der Gesellschaft geführt. Genau diese Situation hat den Putsch für die reaktionären Offiziere und Generäle unausweichlich werden lassen, weil die Disziplin in der Truppe, die vorher erhalten werden konnte, weil das Militär lange Zeit an den gesellschaftlichen Prozessen kaum beteiligt war, weil diese Disziplin in der Truppe nachließ und Matrosen und Soldaten klar zu erkennen gaben, daß sie sich in einer Putschsituation nich jedem Befehl beugen würden. Davon zeugen Interviews mit Soldaten, Unteroffizieren u. a, dafü zeugt aber vor allem der heftige Widerstand, der im Gegensatz zu dem was die Presse hier schreibt, von einzelnen Einheiten der Armee und auch der Marine nach dem Putsch geleistet worden ist. Ůber Tage ist, nach dem was wir aus Santiago jetzt wissen, von Einheiten, auch der Polizei, heftiger Widerstand geleistet worden und z.T. sind ganze Einheiten direkt vernichtet worden, wenn sie sich weigerten, den Befehlen der Putschisten zu gehorchen.

Ein anderer Teil des Widerstandes ist der der Arbeiter, der ganz klar zeigt, daß die Arbeiterschaft noch ne so bewuBt wie heute und auch noch nie so bewaffnet war wie heute. Der Vorwurf, der der Regierung Allende gemacht wird in Bezug auf die angebliche Entwaffnung der Arbeiter, bezieht sich auf das Waffenkontrollgesetz, das 1972 von dem mehrheitlich oppositionellen Kongreß verabschie det wurde. Heute wird behauptet, die Regierung Allende hätte dieses Waffenkontrollgesetz begrüßt. Die Wahrheit ist, daß Allende gemäß seinen präsidentiellen Vollmachten ein Veto gegen dieses Waffenkontrollgesetz vorbereitet hatte und daß innerhalb der Regierungsbürokratie ein Bürokrat den Termin, bis zu dem dieses Veto eingelegt werden mußte, verschlampt hat, wobei nicht offen klar ist, ob es sich da nicht um Sabotage handelt. In der schwierigen Situation Ende Oktober 1972 blieb unter diesen Umständen der Regierung nichts anderes übrig, als das Waffenkontrollgesetz zu verabschieden. Das Militär hat dann acht Monate gewartet, bis es mit der systematischen Anwendung dieses Gesetzes zur Kontrolle von Waffen in Privatbesitz begonnen hat, und zwar einseitig nicht etwa gegen die faschistischen Trupps, die Hunderte von Terrorakten organisiert haben, sondern gegen die Arbeiter in Fabriken, in Wohnungen und sogar auf Friedhöfen.

Wenn man akzeptiert, was du sagst, daß nämlich im Jahr 1970 eine politisierte und mobilisierte Arbeiterklasse nicht vorhanden war die zur Bewaffnung bereit gewesen ware, so kann man aber doch feststellen, daß zu den Wahlen im Marz 1973 ein Hohepunkt der Mobilisierung erreicht war, die in den Monaten danach wieder nachließ. Hatte nicht Allende zu diesem günstigen Zeitpunkt unmittelbar nach den Wahlen die Machtfrage stellen sollen, statt zu warten, bis sie ihm von der anderen Seite zu einem für ihn ungünstigen Zeitpunkt aufgezwungen werden würde, wie es nun tatsachlich geschehen ist?

Die Frage enthält, meine ich, eine Reihe von Hypothesen, die nicht alle richtig sind. Die Frage nach der richtigen Ausnutzung der politischen Konjunktur kann wohl nur 
sinnvoll beantwortet werden, wenn man immer zugleich sowohl die quantitative wie auch die qualitative Dimension der Mobilisierung auf beiden Seiten des Klassenkampfes sieht. Es kommt also darauf an, gleichzeitig zu fragen, wie breit die Mobilisierung und Organisation ist und wofür sie sich mit welchen Mitteln einsetzt. Dann allerdings gewinnt die Frage nach dem richtigen Zeitpunkt fur die Stellung der Machtfrage eine erhebliche Bedeutung.

Nehmen wir uns nun einzelne Monente vor, so ist zunächst zu betonen, dafs zu den Wahlen von 1970 die Arbeiterklasse durchaus politisiert und mobilisiert war, aber wofür? Für die Durchsetzung konkreter ökonomischer, sozialer und politischer Interessen im Rahmen und mit den Mitteln der bürgerlichen Demokratie. Dafür erschien die Eroberung der Regierung als ein entscheidender Schritt, und die Regierung hat ja dann auch die ökonomische, soziale und politische Situation der Arbeiter entscheidend verbessern können. Unter diesen Umständen ist es gar nicht erstaunlich, daß die relativ kleinen Gruppen der Linken, die den bewaffneten Weg als Alternative vorschlugen, nur eine sehr geringe Resonanz hatten.

Die Machtfrage zu stellen wäre dann erstmals im April 1971 möglich gewesen. Am qualitativen Charakter der Mobilisierung hatte sich bis dahin zwar kaum etwas geändert; wie aber die Ergebnisse der Gemeindewahlen vom 4. April 1971 zeigten, hatte sich die Wählerbasis der Unidad Popular so verbreitert, daß sie für einen Moment über eine knappe absolute Mehrheit verfügte. Ein verfassungsänderndes Plebiszit in diesem Moment hätte unter diesen Umständen die Macht der Opposition, zumindest im Parlament und vielleicht auch im Justizapparat, entscheidend schwächen können. So riskant diese Möglichkeit gewesen sein mag, sie ist nicht ernstlich erwogen werden, und Allende selbst hat das später (im November 1972) als einen der grundlegenden taktischen Fehler seiner Regierung bedauert.

Die Situation im März 1973 war dagegen eine völlig andere. Die Parlamentswahlen bedeuteten höchstens quan titativ einen (relativen) Höhepunkt der Mobilisierung der Arbeiterklasse, qualitativ eher das Gegenteil. Im Oktober 1972 hatte die Reaktion erstmals die Machtfrage zu stellen versucht, und darauf hatten die Arbeiter mit einer Mobilisierung auf allen Ebenen geantwortet, die überhaupt erst das Überleben der Regierung Allende sicherte. Die dabei von der Arbeiterklasse entwickelte Energie wurde dann aber in den folgenden vier Monaten in die traditionellen Bahnen des Wahlkampfes gelenkt. Das Wahlergebnis $-7 \%$ mehr für die Unidad Popular als 1970 - war zeifellos ein großer Erfolg für die Linke, gab aber doch nach den Maßstäben, auf die man sich im Wahlkampf selbst eingelassen hatte, keine Legitimationsbasis dafür ab, die Machtfrage in diesem Moment zu stellen, zumal auch die loyalen Militärs nicht bereit waren, sich über die Rolle des Schiedsrichters hinaus zu engagieren. Dazu kam, daß zu dieser Zeit große Differenzen innerhalb der Linken über den einzuschlagenden Weg existierten. Sie waren schon im Wahlkampf deutlich geworden, als die beiden wichtigsten Senatorenkandidaten der Linken ihren Wahlkampf unter so unterschiedlichen Slogans stellten wie ,Nein zum Bürger* krieg!“ (der Kommunist Teitelboim) und „Revolutionäre Entscheidung!“ (der Sozialist Altamirano). Drei Tage nach der Wahl hielt es dann sogar eine Minderheitsgruppe des MAPU für opportun, die Partei zu spalten, wofür sie von den Kommunisten, den Radikalen und der Masse der linken Presse auch noch Beifall und taktische 
Unterstützung erhielt. Kurz, unter diesen Umständen war im März 1973 kaum etwas zu machen.

Es ist nun aber keineswegs richtig zu sagen, daß die Mobilisierung der Arbeiter in den Monaten danach wieder nachließ. Im Gegenteil, besonders der Putschversuch des Oberst Souper fuhrte zu einer Mobilisierung, die breiter und intensiver war als alles, was virher gewesen war. Die Arbeiter besetzten unzählige Fabriken, die Basisorganisationen in den Industriegürteln und die gemeinsamen Kommandos der Linken in den Gemeinden wurden zu einer allgemeinen Erscheinung; die Kommunisten begannen jetzt darm mitzuarbeiten; die taktische Einheit von Sozialisten und Kommunisten wurde betont und gestärkt; überall wurden Verteidigungskomitees gegründet: die Bewaffnung stand von jetzt an für die Arbeiter auf er Tagesordnung.

Wenn sich die Regierung in dieser Situation nicht dazu entschloß, die Machtfrage zu stellen, dann wohl vor allem deshalb weil sich sofort nach der Niederschlagung des Putschversuchs zeigte, daß die loyalen Generäle und Offiziere, entgegen dem vermeintlichen Augenschein, nur noch eine Minderheit der führenden Militärs repräsentierten und darauf drängten, durch einen Ausgleich mit Teilen der Opposition Zeit zu gewinnen und die Putschisten im Militär vorläufig zu neutralisieren. Wie stark sich die rechten Militärs bereits fühlten, sieht man daran, daß sie bereits drei Tage nach dem Putschversuch mit den brutalen Waffenrazzien gegen die Organisationen der Arbeiterklasse begannen.

Tatsächlich bestand also 1970 eine viel begrenztere Ausgangssituation. Es zeigt sich auch hier wieder einmal, daß die Massen der Abhängigen sich über ihre Situation klar werden nicht infolge von Agitation. Verkündung des richtigen Standpunkts usw., sondern daß die massenhaften Bewußtseinsbildungsvorgänge stattfinden aufgrund ganz realer, oft schmerzlicher Erfahrungen. Im Fall von Chile also aufgrund des tatsächlichen Angriffs der Rechten auf die reale Lebenssituation, wie du sagst. 1970 stand der Prozeß der Bewußtseinsbildung erst an seinem Anfang. In der Arbeiterschaft waren Reformhoffnungen noch sehr verbreitet. Welche Möglichkeiten bestanden mun angesichts dieser beschrankten Situation, hinter dem Schutz der Linksregierung die eigenen Strukturen auszubauen und das Kräfteverhältnis zu verbessern, eben zum Teil jenseits oder unterhalb der eigentlichen Politik und der militärischen Macht, und wie weit sind dann solche Möglichkeiten auch tatsächlich ausgenutzt worden?

Man muß wohl zuerst sehen, daß die Eroberung eines Teils der Macht im Staat, eben nur der Regierung - daneben existierten ja noch das oppositionelle Parlament, die reaktionäre Justiz und das Militär, von dem man sehr wenig wußte -, durchaus nicht nur die reformistischen Hoffnungen bestimmter Teile der Arbeiterschaft stärkte, sondern wegen der Maßnahmen, die diese Regierung in Angriff nahm, durchaus auch das revolutionäre Bewußtsein steigern konnte, in dem Sinne nämlich, daß den Arbeitern klar vor Augen geführt wurde: man kann den Imperialismus bekämpfen, die Kupferminen können enteignet werden, die monopolistischen Unternehmen können in die Hand des Staates gebracht werden, der Hunger ist keine Naturnotwendigkeit, das Land kann in die Hände der Bauern schnell und zügig übergehen. Das haben die Arbeiter und Landarbeiter sehr schnell gelernt. 

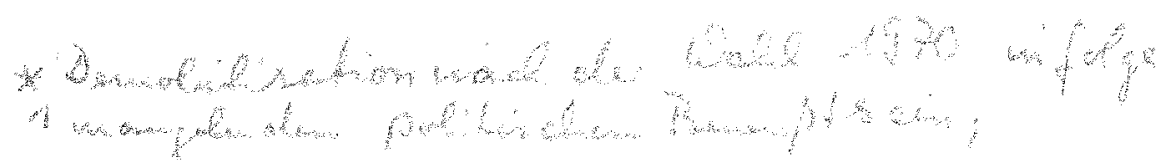

Aber in dem Maße, in dem die Regierung zumindest in den ersten zehn Monaten Erfolge hatte, geschah gleichzeitig eine gewisse Demobilisierung in dem Sinne, daß die Komitees der Unidad Popular, der Volkseinheit, die für die Waht gegründet worden waren, sich auflösten, und außer den von oben eingeführten Bauernräten z. B. kaum neue Organisationsformen der Arbeiterschaft entstanden. Die Versuche, durch politische Überzeugungsarbeit dem abzuhelfen und die Massen stärker zu mobilisieren, waren durchaus sehr begrenzt. Es gab Besetzungen von Fabriken, es gab Besetzungen von kleineren und größeren Landgütern, die der Agrarreform vorgriffen, aber es entstand doch kein allgemeines Bewußtsein von der notwendigen Konfrontation, auf die man sich vorzubereiten hätte.

Wir können daraus lernen, daß nicht der Wille der bewußsten Revolutionäre allein entscheidend ist, sondern auch die Situation, der sich die Arbeiter gegenüberstehen. In dem Maße, in dem wirtschaftliche Schwierigkeiten, auf deren Ursachen man zu sprechen kommen müßte, ein traten und das Kleinbürgertum und die Mittelschichten zur Regierung der Volkseinheit in eine immer schärfere Opposition traten, in dem Maße also, in dem der Klassenkampf von der anderen Seite konzentrierter geführt wurde, in diesem Maße erst organisierten sich die Arbeiter und in diesem Maße auch hatte die Arbeit der politischen Linken innerhalb und außerhalb der Regierung standig mehr Erfolg, soweit sie darauf gerichtet war, das Bewußtsein der Arbeiter zu festigen, die Arbeitermassen zu mobilisieren und sie zu organisieren. Voraussetzung für die Möglichkeit der Mobilisierung und Organisation war aber, daß die Arbeiter wußsten, wofür sie kämpften und welche lirrungenschaften sie verteidigen. Und das hatten sie seit 1970 erfahren.

Das ist eine interessante Präzisierung der Fragestellung; man kann eben nicht einfach mit einer linearen Mobilisierung oder Nichtmobilisierung rechnen, sondern der Prozeß der Bewußtseinsbildung hängt sehr konkret von den jeweiligen Situationen $a b$. Trotzdem möchte ich einen Teil der Frage von vorhin noch einmal wiederholen: Was waren die konkreten Formen dieser Mobilisierung etwa unter der Arbeiterschaft im einzelnen - gerade darüber ist ja hier in der Presse so gut wie überhaupt nichts berichtet worden.

Um ins Einzelne zu gehen: Als die Reaktion auf die Straße ging, zuerst im November 1971 bei dem Marsch der Kochtöpfe, dann aber mit größerem Erfolg im April 1972, als sich zum ersten Mal alle Parteien der Opposition zu einer gemeinsamen Massenkundgebung gegen die Regierung fanden, da gingen auch die Arbeiter auf die Straße, um die Regierung der Unidad Popular zu unterstiitzen. Das war eine kurzfristige Mobilisierung, die nur die Kräfte zeigte, aber die doch zum Bewußtsein und zur besseren Organisation kaum etwas hinzufügte. Doch als im Oktober 1972 die Fuhrunternehmer streikten, der Einzelhandel sich anschloß, die Verbände von Ärzten, Architekten, Rechtsanwälten u. a. versuchten, das gesamte wirtschaftliche Leben lahmzulegen, da wurden die Arbeiter durch die praktische Situation gezwungen, Organisationen zu schaffen, die der Verteidigung der Produktion, der Organisation der Versorgung und dem politischen Kampf dienen sollten. Organisationen, die nicht von der bisherigen Struktur der Parteien und der Gewerkschaften bestimmt waren, sondern die, eher vergleichbar mit den Räten, auf konkrete Situationen

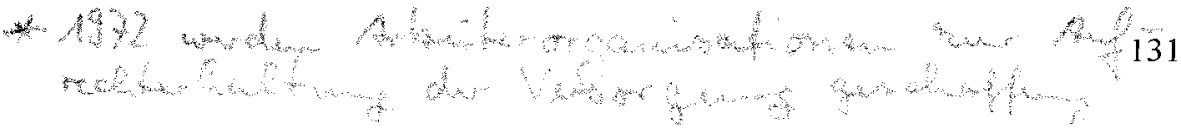


antworteten. Diese Organisationen in den Industriegürteln und ihre Zusammenfassung mit anderen Organisationen der Slumbewohner, der Frauen, der Nachbarschaften und vieler Vereine, haben nach dem Oktober einen gewaltigen Aufschwung genommen und die Verteidigung der Fabriken besonders nach dem Putschversuch vom 29. Juni 1973 systematisch vorbereitet.

Könntest au vielleicht doch wenigstens an einem Beispiel mal zeigen, was der konkrete Inhalt dieser organisierten Tätigkeiten gewesen ist, welche Formen der Zusammenarbeit überregional oder mit anderen ähnlichen Organisationen dabei gefunden worden sind?

Am Stadtrand von Santiago gibt es verschiedene Industriegürtel, innerhalb derer Fabriken, die sehr verschiedene Dinge produzieren, liegen und um die herum sowohl Stadtteile und Elendsviertel als auch ländliches Gebiet liegen. In dem Augenblick, in dem die Versorgung ernsthaft gefährdet wurde und die Unternehmer versuchten, die Arbeiter zu bestechen, damit sie sich an dem allgemeinen Streik, der von den Fuhrunternehmern ausgerufen war, beteiligten, in diesem Augenblick war es eine unmittelbare Notwendigkeit, die Produktion aufrechtzuerhalten und sich gegen Sabotageakte zu schützen. Das war die Hauptaufgabe, die sich die Arbeiter in den verschiedenen Fabriken stellten und zu denen sie sich in Räten organisierten. Die zweite Notwendigkeit war, direkte Formen des Austauschs und der Versorgung zu schaffen, mit denen das Überleben der Bewohner der Stadt und besonders der Elendsviertel garantiert werden konnte; dafür zu sorgen, daß die Güter, die in den Fabriken produziert wurden, an die Bauem und die städtische Bevölkerung kamen und daß die Lebensmittel vom Land an die Arbeiter kamen. Dadurch wurde praktisch auf einem Sektor, z. B. in dem Industriegürtel Cerrillos-Maipú mit den darumliegenden Stadtvierteln Maipú, Cerrillos, aber auch auf dem Land bis hin nach Melipilla, das Gesetz des kapitalistischen Marktes wenigstens zeitweise außer Kraft gesetzt. Aber ähnliches geschah in sehr vielen anderen Gegenden; und in dem letzten Fuhrunternehmerstreik direkt vor dem Putsch, der ja vieí länger dauerte als der Oktoberstreik, haben diese Organisationen sich so stark bewährt, daß für die Masse der Bevölkerung die Folgen des Streiks wesentlich weniger zu spüren waren als im Oktober 1972.

Könntest du noch etwas dazu sagen, wie die Räte sich gebildet haben, wie sie sich zusammensetzten, wie ihr Verhältnis zur Unidad Popular war und wie umgekehrt die verschiedenen Parteien der Unidad Popular sich zur Arbeit der Arbeiterräte verhielten, also sie unterstützten, tolerierten oder auch behinderten?

Wie schon gesagt, war die Bildung von A rbeiterorganisationen neuen Typs in den Industriegürteln und von umfassenderen Kommandos in den Gemeinden der Versuch, für sehr konkrete Probleme eine Lösung zu finden. Natürlich bedurfte es jeweils der Initiative von politisch denkenden Arbeitern oder Gruppen, die im entscheidenden Moment die notwendige Erfahrung und Führungskapazität einbrachten. Wenn ich es recht sehe, waren hier bestimmte Gruppen aus dem MAPU und der Sozialistischen Partei von großer Bedeutung, und zwar die Gruppen des MAPU mehr wegen ihrer die Situation des Moments aufgreifenden Initiativen, die Soziali- 
sten vor allem wegen des Gewichts, das ihre Teilnahme bedeute te.

Um das klarer zu machen, will ich ein wenig ins einzelne gehen. Im Mai 1972 fand in Conepción auf Initiative des lokalen MAPU eine "Volksversammlung" statt, in der zum ersten Mal die Organisation der Linken von der Basis her im Mittelpunki stand. Obwohl außer den Kommunisten alle lokalen Organisationen der Linken an dieser Versammlung teilnahmen, wurde diese Initiative von Allende und den nationalen Führungen der wichtigsten Parteien (einschlieklich des MAPU und der Sozialistischen Partei) mehr oder weniger scharf kritisiert, wobei mit dem Vorwurf des Anarchismus und des ultralinken Sektierertums nicht gespart wurde. Im Juni 1972 organisierten sich dann zum ersten Mal die Arbeiter mehrerer Fabriken eines Industriegürtels (nämlich des schon erwähnten von Cerrillos-Maipú), um gegen die Verhaftung von Landarbeiterführern des MAPU durch die reaktionäre Justiz und gegen die Absetzung des sozialistischen Innenministers durch das oppositionell beherrschte Parlament zu protestieren. Die dort gemachten Erfahrungen erlaubten dann im Oktober 1972 unter dem Druck des Unternehmerstreiks die schnelle Übernahme dieser Organisation sform auch in anderen Industriegürteln.

Die alle Formen der Basisorganisation in einer Formel zusammenfassende Losung ,Volksmacht schaffen!" ist meines Wissens zum ersten Mal Anfang Dezember 1972 vom MAPU, der sich gerade eine neue Führung gegeben hatte, systematisch verbreitet worden. Sie wurde dann sehr schnell vom MIR, von der Christlichen Linken und Teilen der Sozialistischen Partei aufgegriffen. Erst im Juni 1973 wurde sie zur Losung der gesamten chilenischen Linken.

In die Führung der Basisorganisationen wurden natürlich die aktiven Arbeiter gewählt, die das Vertrauen ihrer Kollegen hatten. Das waren oft, aber nicht notwendig, die Sprecher der Gewerkschaften, die ihrerseits nach parteipolitischen Gesichtspunkten gewählt worden waren. Man darf aber nicht vergessen, dak auch zu diesem Zeitpunkt noch viele Arbeiter nicht gewerkschaftlich organisiert waren und viele Kleinbetriebe gar keine gewerkschaftliche Organisation kannten. Insofern war, wenigstens nach dem Konzept, die Organisation in den Industriegürteln viel umfassender als die Gewerkschaftsorganisation. Auf der anderen Seite war sie auch umfassender als die Unidad Popular, weil sich der MIR von Anfang an daran beteiligte und weil das Bestreben überall dahin ging, auch die unabhängigen und christdemokratischen Arbeiter einzuschließen, was auch in wachsendem Umfang gelang. Weil sich in der Führung das Kräfteverhältnis der politischen Parteien innerhalb der beteiligten Arbeiterschaft spiegelte, lag die Führung der Organisationen fast ausnahmslos bei Mitgliedern der Sozialistischen Partei.

Der MIR hat diese räteähnlichen Organisationen von Anfang an unterstützt, weil er sich von ihnen eine Stärkung der revolutionären Kräfte gegen die von ihm als „reformistisch" bekämpften Tendenzen in der Regierung und besonders in der Kommunistischen Partei versprach. Das Selbstverständnis der meisten Organisationen ging aber eher dahin, unter Betonung der eigenen Autonomie in Einzelfragen, die Regierung gegen den Angriff der Rechten zu stärken und zu stützen und dem Zentralen Gewerkschaftsbund mit seiner traditionellen und teilweise bürokratischen Struktur neue Impulse zu geben.

Den Kommunisten war selbst das lange Zeit zu viel. Nach dem Oktober 1972 , 
als sie beim Autbau der Organisationen beteiligt gewesen waren, zogen sie sich systematisch wieder zurück, weil sie von den Industriegürteln und Gemeindekommandos eine den Gesamtprozeß gefährdende unkontrollierbare Gegenmacht gegen die Regierung und eine Konkurrenz zu den häufig von Kommunisten geführten Gewerkschaften fürchteten. Erst als nach den Wahlen vom März 1973 der Druck der Rechten wieder stärker wurde, zeigte sich die Parteiführung wieder stärker bereit, dem Druck der eigenen Basis nachzugeben und die Mitarbeit der Kommunisten in den Organisationen der Volksmacht zu fördern. In den letzten Wochen vor dem Putsch war es also dann endlich so weit, dak die gesamte Arbeiterklasse daran war, sich über die Grenzen der Unidad Popular und der Gewerkschaften hinaus gemeinsam zu organisieren.

Du hast vorhin die Frauen erwähnt, die mit Kochtöpfen auf die Straßen gegangen sind. Man vermutet, obwohl das hier in der Presse nicht gesagt worden ist, daß diese Frauen den besseren und besten Schichten angehörten. Aber zunächst einmal paßt es in das gängige Bik von konservativen Tendenzen bei den Frauen überhaupt; es hat ja z. B. 1919 zur groß3en Überraschung der SPD ein beträhtlicher Teil auch der Frauen der Arbeiterschaft rechts gewählt, jedenfalls nicht so hinks wie erwartet worden war. In Chile haben nun aber offenbar auch die Frauen auf der Linken eigenständige Formen von Organisationen gebildet. Was waren die konkreten Interessen, die die Franen mobilisiert haben, und zwar nach links hin mobilisiert haben?

Die Frauen haben unter den Schwierigkeiten, die durch das Wirtschaftschaos ausgelöst wurden, wahrscheinlich mehr zu leiden gehabt als die Männer, weil sie gezwungen waren, oft Stunden und Stunden nach Waren anzustehen, um das Essen für die ganze Familie zu organisieren. In dieser Situation war die Antwort, die die Frauen gaben, eine Antwort, die sich ganz klar an den Klassengrenzen orientierte. Die Arbeiterfrauen wurden sich schnell klar darijber, wer es war, der ihnen das Leben schwermachen wollte. Sie hatten zum ersten Mal Geld und konnten kaufen, wenn auch nur unter den Schwierigkeiten, die das Schlangestehen mit sich bringt. Die Frauen der Oberschicht, die die Demonstration der Kochtöpfe organisiert hatten, hatten inmer Geld gehabt, und für sie war die Regierung schuld daran, daß sie jetzt zum ersten Mal nicht alles sofort kriegten, was sie gerne haben wollten. Tatsächlich gab es meistens genügend Möglichkeiten, sich auf dem von den besseren Schichten organisierten Schwarzmarkt doch alles zu besorgen, was sie haben wollten.

Du hast eben gesagt, die Arbeiterfraten whilen, wer ihnen das Leben schwermachte. Du hast verwiesen anf die Polarisierning, die da stattgefinden hat. Das führt zu der Frage nach der Rolle der Mittelschichten. von denen man ja hier immerhin verhälmismäßig noch am meisten gehört hat, beispielsweise von den Fuhrunternehmern. die cinen sogenannten Streik veranstaltet haben. Ist die Antwort nun ganz einfach die, das diesc Polarisienng wesentlich von der Stellang in gesellschafilichen Reproduktionsprozej bestimmt worden ist, daß also auf der einen Seite die ablä̈ngig Arbeitenden standen und auf der anderen Seite die großen, kleinen und sogar kleinsten Besitzer von Produktionsmitteh. Handwerker. kkine Geschäftsleute usw.? Oder gab es da gewisse Moglichkeiten der Variation der Entscheidungen nach 


\section{der einen oder anderen Seite hin?}

Die Mittelschichten, für die man - besonders im Englischen und dann auch im Spanischen - oft auch den unglücklichen Ausdruck ,Mittelklasse" verwendet, sind durchaus nicht eine geschlossene Einheit. Natürlich ist es notwendig zu versuchen, diese Mittelschichten zu neutralisieren, wobei zunächst anzumerken ist, daß große Teile des Subproletariats, die nach ihrer Stellung im Produktionsprozeßs vielleicht diesen Mittelschichten zugerechnet werden könnten (wie z. B. Gelegenheitshändler, Schuhputzer, Kleinsthandwerker, Wäscherinnen usw.) in der Situation eines unterentwickelten Landes wie der Chiles nicht zu den reicheren, sondern zu den armen Schichten gezählt werden müssen und deshalb von dem wirtschaftlichen Chaos, das geschürt wurde, genauso betroffen wurden wie die Arbeiter selbst und deshalb vielfach an die Seite der Arbeiter gefunden haben.

Aber beschäftigen wir uns mit den eigentlichen Mittelschichten, $z u$ denen ja neben den Besitzern von Produktionsmitteln auch die Freiberuflichen gehören und eine ganze Zahl von relativ privilegierten Angestellten und Technikern, auch Staatsangestelite wie Lehrer und nicht zuletzt auch die Berufsmilitärs und die Polizei. Es ist nun zweifellos nicht so, dafs alle diese Mittelschichten voll auf der Seite der Rechten standen, aber es ist auch eine Illusion anzunehmen, dath in der Koalition, die die Unidad Popular darstellte, die Mittelschichten eine sehr breite politische Unterstïtzung gaben. 1970 hat latsächlich nach einer genaueren Analyse der Wahlergebnisse nur ein sehr schr geringer Teil der Mittelschichten für die Unidad Popular gestimmt, und der Stimmenzuwachs, den die Unidad Popular nach 1970 bei Wahlen erzielt hat, ist eher aus dem Subproletariat gekommen als aus den Mittel. schichten.

Nun muß man auch eine Unterscheidung machen zwischen politischer Unterstützung auf der einen Seite und wirtschaftlichem Wohlverhalten auf der anderen. Bei klarer Erkenntnis der Situation hätte die Unidad Popular sich nicht notwendig durch gewisse Verluste von politischer Unterstïtzung durch die Mittelschichten (etwa am Anfang des Jahres 1972) dazu treiben lassen müssen, den Mittelschichten hinterherzulaufen, wie das dann im Laufe des Jahres 1972 geschehen ist. Die Unidad Popular hat unter maßgeblichem Einflußs des kommunistischen Finanzministers Orlando Millas etwa seit Juni 1972 eine Politik durchgefürt, die darauf hinauslief, dem Kleinbürgertum Garantien zu geben und Konzessionen zu machen und zwar in einer Weise, die bedeutete, dafs die Gesetze des kapitalistischen Marktes wieder stärker zur Richtlinie der Wirtschaftspolitik wurden.

Angesichts verschiedener Gleichgewichtsstörungen des kapitalistischen Marktes, die durch den Angriff des Imperialismus einerseits, durch die vorherigen Maßnahmen der Regierung andererseits schon zutage getreten waren, war nun aber dieser Versuch einer Orientierung an den Gesetzen des kapitalistischen Marktes nur der Auftakt zu einer ungeheuren Spekulation dieser Mittelschichten und des Kleinbürgertums. Sie kümmerten sich nicht, wie man das erwartet hatte, um Produktion und Investition, sondern um spekulative Geschäfte. Nun ist es zweifellos nicht falsch, den Mittelschichten Garantien zu geben, um ihr wirtschaftliches Wohlverhalten zu sichern. Diese Garantien müssen aber verbunden sein mit einer scharfen 
Kontrolle sowohl durch den Staatsapparat wie durch die unmittelbaren einen Prozek in Bewegung, von dem zugleich auch ande re Teile der Mittelschichten, die ihnen gemacht werden, die Überzeugung ableiten sollen, daßs sie den Prozeß beherrschen und sich dann entsprechend wild benehmen.

- Als das Bürgertum und Kleinbürgertum zu spekulieren begann, setzte es einen Prozer in Bewegung, von dem zugleich auch andere Tcile der Mittelschichten, ja sogar Arbeiter und sozialisierte Betriebe erfaßt wurden. Solange keine klare Planung bestand und jeder Betrieb in weiten Teilen darauf sehen mußte, sein Eigeninteresse zu vertreten, waren nicht eimmal nationalisierte Betriebe frei von der Hoffnung, durch Spekulation Fortschritte machen zu können. Viel weniger die Staatsangestellten und Techniker und privilegierte Arbeiter. Die Aussicht, es da, sich um die Produktion selbst noch zu kümmern? In dem Maße, in dem nun als das Ergebnis der Produktion selbst. In dem Maßse, in dem bestinmte Lebensmittel oder sonstige Dinge des täglichen Konsums knapper wurden, konnte man durch Horten und Verkaufen auf dem Schwarzmarkt ungeheure Gewinne machen. Was lohnte sich da, sich um die Produktion selbst noch zu kümmern? In dem Maße, in dem nun klar wurde, daß dem Chaos und der Spekulation tatsächlich nur noch durch Maßnahmen abgeholfen werden konnte, die zu einer Sozialisierung sowohl der Produktion als auch des Handels in immer stärkerem Ausmaßhe führten, teil te sich die Gesellschaft immer klarer in die, die dieses als mögliche Lösung zu akzeptieren bereit waren, nänlich vor allem die Arbeiter und die ärmere Bevölkerung, und die, die darin nicht nur einen Verlust ihrer Spekulationsmöglichkeiten sahen, sondern auch ihrer bisherigen wirtschaftlichen ,Freiheit“. Unter diesen Umstanden ist es eher erstaunlich, dat bestimmte Teile der Mittelschichten, wie z. B. große Teile der Volksschullehrer, aber auch der Professoren, Ärzte und sogar Fuhrunternehmer, sich nicht auf die Seite der Rechten geschlagen haben, sondern die Regierung der Unidad Popular weiter unterstützten.

Die Lehre, die man daraus wahrscheinlich ziehen könnte, ist, daß ohne eine frühzeitig einsetzende gesellschaftliche Planung unter Einfluß der Arbeiterschaft und ohne eine gesellschaftlich ausgeübte Kontrolle Teile der Mittelschichten bereit sind, das Chaos durch die Vertretung ihrer unmit telbarsten Interessen zu vergrößern und dalk sie die dann notwendigen Schritte zur Überwindung der Situation so fürchten, daß sie sich auf die Seite des Klassengegners fäst notwendig schlagen müssen.

Gegen diese von dir eben gezogene Folgerung könnte man allerdings einwenden, daß sie aus objektiven Gründen gar nicht zulässig ist. Denn ein beträchtlicher Teil des gesellschafilichen Reproduktionsprozesses ist ja gerade in der Hand dieser kleinen Warenproduzenten usw.; das schlagendste Beispiel sind doch wohl diese Fulhrunternelmer, die den größzten Teil des Transportsystems in ihrer Hand haben. Das Transportsystem z. B. war eben einer Planung nicht zugänglich. Dic Frage wäre also: Ist das nicht eine leere Enpfehhng, die jedenfalls unter den spezifischen Verhälnissen in Chile undurchfüllrbar gewesen wäre? Ich muß elarlich sagen, diese Empfehhung erimert mich etwas an die verbreitete Losung von der Zerschlagung des Staatsapparates, bei der man sich dann immer fragt, wie sich das die Leute 
eigentlich vorstellen.

Natürlich wird man nicht annehmen können, daß der Imperialismus und die Bourgeoisie darauf verzichten, so große Teile des Kleinbürgertums wie immer möglich für ihre Zwecke einzusetzen, und dafür auch bereit sind, beliebige Mittel zur Verfügung zu stellen. Das war in Chile gar nicht so kostspielig, wie man sich das vielleicht vorstellen mag. Aber die Frage ist ja nicht, wie man einen Angriff der Rechten verhindern kann - das wird man wohl nie können -, sondern die Frage ist, wie man verhindern kann, daß dieser Angriff zum Erfolg führt und sich wie in Chile auf eine Massenbewegung der Mittelschichten und besonders des Kleinbürgertums stützen kann. Hätte frühzeitig der Versuch bestanden, tatsächlich gesellschaftlich zu planen und keine Konzessionen zu machen, ohne gleichzeitig zu kontrollieren, was das Kleinbürgertum mit diesen Konzessionen anfängt, dann wären die Angriffsmöglichkeiten der Rechten sehr viel beschiänkter gewesen. Aber das verschiebt nur die Frage, denn eine Planung, die die Privatwirtschaft mit einbezieht und nicht bei indikativer Planung stehenbleibt (also bei Globaldaten, nach denen sich dann die Privatunternehmer richten können oder auch nicht), verlangt ein Maß von Arbeit auf seiten der Planer und der Arbeiter, die die Inhalte dieser Planung zu bestimmen hätten, wie man sich das kaum vorstellen kann; eine solche Planung verlangt vor allem auch das Bewußtsein von der Notwendigkeit dieser Planung und Kontrolle. Dieses Bewußtsein war in Chile sehr wenig entwickelt in den Jahren 1971 und 1972 und ist erst 1973 unter dem Eindruck der Probleme sehr viel weiter entwickelt worden, so daß in den letzten Wochen und Monaten außerordentlich intensiv an einer sehr detaillierten Planung für das Jahr 1974, besonders für den gesamten Industriebereich, gearbeitet wurde.

Die Forderung nach detaillierter Planung scheint mir zunächst genauso abstrakt zu sein wie die Vorstellung, daß man sich im Staatsapparat in der Position befindet, die Mittelklassen zu ökonomischem Wohlverhalten zwingen zu können. Wenn ich die chilenische Situation aus deinen Äußerungen richtig verstanden habe, befand sich doch die Linke immer noch im Vorfeld der realen Übernahme der politischen Macht. Die Möglichkeit, Zwischenklassen zu ganz bestimmtem ökonomischem Verhalten zu zwingen, würde ja voraussetzen, daß die Machtfrage entschieden ist; daher ergaben sich ja auch die Schwierigkeiten der Unidad Popular, erfolgreiche gesellschaftliche Bahnen überhaupt zu betreten.

Tatsächlich verfügte die Unidad Popular nur über einen begrenzten Teil der Macht und nicht über die ganze politische Macht. Aber es ist doch zu fragen, ob nicht bei einer größeren Ausnutzung der Möglichkeiten, die sie hatte, der Möglichkeiten der Kontrolle und der Planung, das von den Mittelschichten mitgetragene Chaos in der Wirtschaft stärker hätte eingedämmt werden und damit der politische Zusammenbruch vermieden oder hinausgeschoben werden können. Im ersten Jahr der Regierung Allende gab es durchaus das, was wir vorhin wirtschaftliches Wohlverhalten der Zwischenschichten genannt haben; die Inflationsrate sank sogar. Erst in dem Augenblick, in dem wirtschaftliche Schwierigkeiten auftraten, begann die Spekulation, begann das Kleinbürgertum zu rebellieren und an der Zerstörung der Wirtschaft mitzuarbeiten. Man muß zugeben, daß die Kontrolle durch den Staatsapparat sehr 
erschwert war, einmal weil dieser Staatsapparat nicht für eine solche Aufgabe geschaffen war (es waren im wesentlichen bürgerliche Institutionen, Institutionen des bürgerlichen Staates) und zum anderen, weil große Teile des Staatsapparates von Gegnern der Regierung besetzt waren. Es ist aber zu fragen, ob sich die Regierung der Unidad Popular aus Rücksicht auf die Interessen der Mittelschichten so weit hätte treiben lassen sollen, daß sie Formen der Arbeiterkontrolle, wie sie von der Linken z. T. gefordert wurden, ausschloß, wie sie das getan hat. Erst unter dem Eindruck des Putschversuches vom Juni 1973 und des zweiten Fuhrunternehmerstreiks sind wirklich alle Formen der Arbeiterkontrolle von der Unidad Popular gutgeheißen und gefordert worden.

Es ist in jedem Fall eine schwierige Sache, hier von der Ferne aus die Frage zu stellen, $o b$ in Chile von der Unidad Popular grundsätzliche Versäumnisse gemacht worden sind. Gerade angesichts dessen, was jetzt jeden Tag dort in Chile passiert, glaube ich aber doch, daß wir im Hinblick auf das, was wir für uns lernen können, diese Frage doch stellen sollten. Die Frage also, ob über das was bisher schon angeschnitten worden ist hinaus, von grundsätzlichen Versäumnissen gesprochen werden muß?

Ich glaube, daß die Grundversäumnisse erstens darin lagen, daß man das Militär zwar an die Regierung zu binden suchte, sich aber nicht gleichzeitig systematisch genug darauf vorbereitete, bei einem eventuellen Verfassungsverrat des Militärs die notwendige Gegenkraft zu haben; und zweitens darin, daß man es nicht verstanden hat, die Mittelschichten so zu kontrollieren, daß sie zu einem spekulativen und damit vollkommen destruktiven Verhalten keine Gelegenheit fanden.

Darüber hinaus sind natürlich viele andere Fehler im einzelnen gemacht worden. Etwa, daß man an Punkten angegriffen hat, an denen man noch hätte warten können, d. h. daß man in manchen Fällen den Faktor Zeit nicht richtig in Betracht gezogen hat, oder auch, daß man an Punkten nicht angegriffen hat, an denen man schon viel früher hätte attackieren müssen. Um ein Beispiel zu nennen: Der Versuch, die Schule auch nur in einem demokratischen Rahmen zu reformieren und bestimmte Privilegien abzuschaffen, die für das Bürgertum in dem gegenwärtigen Schulwesen vorhanden waren, hat nichts eingebracht außer einem zusätzlichen Argument für das Bürgertum, daß sich in dieser Auseinandersetzung sogar auf einen Teil der Kirche stützen konnte. Das hätte noch mehr Zeit gehabt. Im ganzen ist die Unterstützung, die für die Unidad Popular aus dem Subproletariat von Anfang an hätte kommen können und dann auch tatsächlich gekommen ist, unterschätzt worden. Das liegt daran, daß die bestimmenden Parteien der Unidad Popular, also die Sozialistische Partei und die Kommunistische Partei, im wesentlichen Arbeiterparteien waren, die als Bündnispartner immer nach den sog. Mittelklassen suchten und darüber vergaßen, daß es eine große Zahl von Menschen gibt in Chile, die in sehr elenden Verhältnissen leben. Parteien, die dann zur Unidad Popular gestoßen waren, wie etwa der MAPU, haben sich zwar dann in stärkerem Maße auch auf die Landarbeiterschaft stützen wollen; aber es ist bezoichnend, daß in den Elendsvierteln die Arbeit gegen die Demagogie der Christdemokratie weniger aus diesen Parteien kam, erst recht nicht aus der Radikalen Partei, sondern aus der Christlichen Linken, die 
bewußt sich nicht als marxistische Partei verstand, und aus dem MIR, der außerhalb der Unidad Popular stand. Diese Unterschicht hat einmal in Chile für die Wahlen eine größere Bedeutung als in Europa; und gerade die Zunahme der Wählerschaft der Unidad Popular hat ja die Putschisten so sehr besorgt gemacht, daß sie zuschlagen mußten. Andererseits mußte aber auch ein Weg gefunden werden, die Rekrutierungsphase faschistischer Organisationen, die immer nach dem Lumpenproletariat als erstem Verbündeten oder besser Söldner suchen, zu reduzieren. Das ist in einem erstaunlichen Maße gelungen, wenngleich die kleine faschistische Organisation Patria y Libertad immer noch Leute gefunden hat, die sich für einige Groschen zur Verfügung gestellt haben, um irgendwo zuzuschlagen. Die Hauptmasse der Leute, die bei Patria y Libertad arbeiteten, kam aber aus den reichen Schichten, was man etwa ablesen kann an den Listen der Verhafteten, die Attentate und Sabotageakte unternommen hatten.

\section{Wer stand denn nun eigentlich direkt hinter dem Putsch?}

Die Bourgeoisie, vor allem vertreten durch die Nationale Partei, hat zweifellos schon von Anfang an nichts unversucht gelassen, um der Regierung Allende so schnell wie möglich ein Ende zu bereiten. Und nicht nur die ITT-Affäre von 1970 verrät, daß die in ternationalen Konzerne dieselbe Strategie verfolgten und der Regierung Allende so früh wie möglich ein schnelles Ende wünschten und auch daraufhin gearbeitet haben. Im Jahre 1973 hat sich dann aber für die Christdemokraten, zumindest für ihren rechten Flügel, der von dem ehemaligen Präsidenten Frei beherrscht wird, herausgestellt, daß ihre bisherige Strategie (die sog. Strategie der russischen Marschälle, die darauf hinauslief, den Gegner bis kurz vor Moskau kommenzulassen, um ihn dann zu schlagen) keine Chance mehr hatte. Es war bei dem Anwachsen der Anhängerschaft der Regierung Allende auch unter den Soldaten nicht mehr zu erwarten, daß die Rechte ohne weiteres in den nächsten drei Jahren auf unblutige Weise die Macht wieder erobern könnte, und selbst für 1976, für die nächsten Präsidentschaftswahlen, lagen die Chancen schlecht. In dieser Situation hat der rechte Flügel der Christdemokratie und besonders Eduardo Frei alles getan, um den Putsch zu fördern. Daß jetzt auch die Christdemokratische Partei verboten ist, widerlegt das nicht. Untersucht man den Verlauf des Putsches im einzelnen, so erkennt man, daß zu Anfang versucht wurde, jede Möglichkeit für eine scheindemokratische Lösung nachher offenzulassen. Aber die Konterrevolution hat ihre eigene Logik und ihre eigene Dynamik. Die Putschisten, selbst wenn sie wie der General Pinochet an der Spitze der Junta bisher nicht als Vorkämpfer des Faschismus hervorgetreten waren, sind jetzt gezwungen, $T$ ag für Tag mehr brutalen Terror anzuwenden, um dem Widerstand der Arbeiter und eines Teils der Soldaten zu begegnen. Da sie sich gezwungen sehen, den Marxismus auszurotten, um diesen Widerstand zu brechen, müssen sie mit dem Marxismus auch die letzten Formen bürgerlicher Demokratie und Legalität ausrotten, weil die Idee des Sozialismus in Chile die Massen ergriffen hat und das, was sie jetzt erleben, sie nur in dieser Idee bestärken kann. Demokratie, gleich welcher Art, ist in Chile deshalb ohne Marxismus nicht denkbar.

Versuchen wir abschließend, Erfahrungen der Linken in Chile, in der Unidad Popular vereinigt und aufserhalb, zu verallgemeinern (wir meinen natürlich nicht, daß wir 
alle Verallgemeinerungen auf die Situation in Westeuropa bzw. etwa in der BRD für übertragbar halten). Die Erfahrungen der chilenischen Linken können uns helfen, die spezifischen Bedingungen einzuschätzen, die zur Erschüttenung der bürgerlichen Gesellschaft in ihren Grundfesten führen können. Wir konzentrieren uns dabei auf drei Punkte, die bei uns in der Diskussion eine besondere Rolle spielen und wo bestimmte Gruppen z. T. unhaltbare Positionen vertreten.

Eine zentrale Frage bei der Einschätzung dieser Bedingungen ist die Frage nach der Möglichkeit und der Funktion einer Linksregierung in einer Phase, in der vor allem die okonomische Macht der Bourgeoisie noch nicht gebrochen ist. In allen Darstellungen der Arbeit der Unidad Popular - solange sie nicht bloße Diffamierungen sind - wird die in drei Jahren geleistete Mobilisierung und Politisierung der Massen hervorgehoben. Die Betonung dieser Tatsache ist besonders denjenigen gegenüber wichtig, die verkennen, daß bei der Regierungsübernahme der Unidad Popular eine solche Politisierung nicht vorhanden war, die es ihr vielleicht erlaubt hätte - wie eine abstrakte Forderung hierzulande lautet - das Volk zu bewaffnen. Diese Tatsache muß aber vor allem betont werden, um auf die erfolgreiche Moglichkeit hinzuweisen, hinter dem Schutzschild einer Linksregierung die Massen zu mobilisieren und für weiterreichende Ziele zu politisieren und diese Mobilisierung durch Schaffung basisnaher Organisationsformen zu fördern und abzusichern. Für linke Gruppen, die vielleicht schärfer als andere die begrenzte Funktion einer Linksregie. rung als Übergangsregierung sehen, kann und darf es daher keine Kritik einer solchen Linksregierung um jeden Preis geben. Kritik muß immer solidarisch sein und darf nur und muß dann geübt werden, wenn Maßnahmen der Linksregierung die Funktion des Schutzschildes in Frage stellen und die Mobilisierung der Massen behindert wird.

Die Antwort auf die erste Frage des Interviews laßst sicher Verallgemeinerungen $z u$, die überall in Westeuropa zu beherzigen sind. Die Justiz, die staatliche Bürokratie, vor allem aber die Polizei und das Militär stehen immer, so neutral sie sich auch zu geben scheinen, wenn sie sich aus den in der ubrigen Gesellschaft ausgetragenen Auseinandersetzungen herauszuhalten scheinen oder wenn sie daraus herausgehalten werden, in Situationen der Entscheidung auf der anderen Seite. Es gilt deshalb von Anfang an, die durch eine Mobilisierung der Massen sich verschäfenden Widersprüche auch in Polizei und Armee wirksam werden zu lassen. Das wird nur möglich sein, wenn auf eine an Äußerlichkeiten sich festmachende pauschale Verurteilung von Polizei und Armee verzichtet wird zugunsten einer langwierigen Aufklärung und Agitation in Polizei und Armee.

Schileßlich jedoch müssen wir uns die Erfahrung, wie die Regierung der Unidad Popular in Chile ihr Ende gefunden hat, vor Augen halten. Es ist sicher richtig und wichtig, sich in der Agitation immer wieder auf Verfassung und Recht zu berufen; in Wirklichkeit ist es allerdings so, daß da, wo die bürgerlich-parlamentarischen Möglichkeiten für die Durchsetzung einer radikalen Veränderung, ja nur für eine Gefährdung der ökonomischen Machtverhältnisse voll ausgenutzt werden, daß da die Bourgeoisie just diese Verfassung und dieses Recht nur noch als einen Fetzen Papier betrachtet, auf dem sie Soldatenstiefel herumtrampeln läßt. 
Ausgewählte Veröffentlichungen über die Geschichte Chiles und die Regierungszeit der Unidad Popular

Über die Entwicklung der Klassenkämpfe in den Jahren der Regierung Frei und besonders der Unidad Popular unter Allende liegen mittlerweile zahlreiche Bücher und Aufsätze vor, aus denen hier nur einige - u. E. auch nach dem Putsch des Militärs im vergangenen September - besonders brauchbare Arbeiten genannt werden sollen.

Autorenkollektiv: Chile zwischen bürgerlichem Weg und sozialistischer Revolution, erstmals in deutsch erschienen in: Arbeiterpolitik. Informationsbriefe der Gruppe Arbeiterpolitik, Nr. 4-6, Bremen 1971; nachgedruckt in: Probleme des Klassenkampfs, Nr. 3, Mai 1972, S. 145-184.

In diesem Beitrag wird versucht, von den konkreten Bedingungen, wie sie 1970 in Chile bestanden haben, auszugehen und zu diskutieren, welche Möglichkeiten sich durch die Unidad-Popular-Regierung für die revolutionäre Linke eröffnen, ihre eigenen Positionen und Kräfte auszubauen, d. h. hinter dem Schutz einer solchen Linksregierung die Kräfteverhältnisse zu ihren eigenen Gunsten zu verschieben. Die Konzentration auf diese Fragestellung ist für die „Fehler"-Diskussion über die Unidad Popular Chiles, wie sie innerhalb der Linken der BRD geführt wird, besonders wichtig, da es meistens in diesen Einschätzungen nicht gelingt, die allgemeinen Aussagen (wie der Notwendigkeit der Zerschlagung der bürgerlichen Institutionen und der Armee als Bedingung des Aufbaus des Sozialismus) mit den konkreten Ausgangsbedingungen (Bewußtseinsstand der Arbeiterklasse, Rechtsstaats- und Parlamentarismusillusionen, Kräfteverhältnisse zwischen den Klassen) zu verbinden.

Luis Vitale: Que despues de las elecciones? Santiago de Chile 1970.

In ähnlicher Weise versucht auch dieser marxistische Historiker und langjährige Gewerkschaftsführer die Möglichkeiten und Grenzen zur Durchsetzung der Interessen der Arbeiterklasse zu bestimmen, die sich durch den Wahlsieg der Unidad Popular ergeben können. Dieses Buch enthält eine straffe und informative Darstellung der sozialen Prozesse, die zur Niederlage der Christdemokraten in den Wahlen 1970 gefürt haben. Vitale diskutiert in diesem Beitrag ebenso realistisch den Bewußtseinsstand der chilenischen Arbeiterklasse und die realen Kräfteverhältnisse zwischen den Klassen, um von daher die von der revolutionären Linken zu leistenden Aufgaben zu entwickeln.

Dieter Boris/Elisabeth Boris/Wolfgang Eberhardt: Chile auf dem Weg zum Sozialismus, Köln 1971.

Brauchbar ist in diesem Buch, das aus Seminararbeiten an der Marburger Universität hervorgegangen ist, vor allem der historische Teil und die Passagen über die Strategiediskussion zwischen der Kommunistischen und der Sozialistischen Partei. 
Elisabeth Boris/Hans Haustein: Die Unidad Popular und die Offensive der Reaktion in Chile, in: Blätter für deutsche und internationale Politik, Nr. 1/1973.

Dieser Aufsatz bringt präzise Daten und Informationen über das Arsenal der Reaktion, angefangen von den Manipulationen der Kupferpreise und der Zurückhaltung der Kredite bis hin zu den Umsturzplänen und den koordinierten Sabotageakten der Rechtsparteien und faschistischen Organisationen. Ebenso gehen die Verfasser auf die Antwort der in der Unidad Popular zusammengeschlossenen Parteien auf diese Offensive der Konterrevolution ein.

Reinhard von Brunn: Chiles ,gesetzlicher Weg“ - eine Sackgasse? in: Kritische Justiz, Heft 3/1973 (im gleichen Heft weitere Beiträge zu Chile)

geht besonders auf Einzelheiten der „Gegen-Legalität“ der Bourgeoisie und ihre sichere Verankerung in Organen der Justiz und des Rechnungshofes ein.

Hans Rudolf Sonntag: Revolution in Chile, Frankfurt 1972.

Vom theoretischen Rahmen A. G. Franks ausgehend beschreibt Sonntag materialreich die Jahre vor dem Wahlsieg der Linkskräfte 1970. Diese Arbeit enthält einen dokumentarischen Teil mit Interviews, Auszügen aus dem Programm der Unidad Popular sowie der ersten Botschaft Allendes an den Kongreß.

Arno Münster: Chile - friedlicher Weg? Historischer Bericht und politische Analyse, Berlin 1972.

Dieses Buch enthält einen historischen Abschnitt zur Sozialgeschichte Chiles seit Erlangung seiner Unabhängigkeit von Spanien, der jedoch recht flüchtig zusammengestellt ist. Besonders lesenswert sind demgegenüber die Ausführungen über die konkreten Veränderungen in den Produktionsverhältnissen, die Informationen über die sozialen Prozesse an der ,Basis", in denen sich in Form von Arbeiter- und Bauernräten und von Verteilungskomitees usw. die Strukturen und Kräfte einer Doppelherrschaft andeuten.

A. G. Frank: Kapitalismus und Unterentwicklung in Lateinamerika, Frankfurt 1969 ;

Herman Ramirez Necochea: Englands wirtschaftliche Vorherrschaft in Chile, in: Lateinamerika zwischen Emanzipation und 1mperialismus, Berlin (DDR) 1961.

J. Fred Rippy: Latin America. A Modern History, New York 1958.

Zur Geschichte Chiles seit Erlangung seiner politischen Unabhängigkeit von Spanien seien aus den zahlreichen Publikationen nur o.g. drei Arbeiten erwähnt. In diesen Büchern wird beschrieben, wie die Eingliederung dieses Landes in den Weltmarkt aus den Erfordernissen des gesamtgesellschaftlichen Reproduktionsprozesses der aufsteigenden imperialistischen Industrienationen erfolgt ist und wie diese auch politisch erzwungen wurde. Die Kenntnis der jeweiligen geschichtlichen Stellung der Länder der Dritten Welt innerhalb der gesamten Reproduktionszusammenhänge auf dem Weltmarkt muß in die Diskussion der Schwierigkeiten des Übergangs zum Sozialismus in unterentwickelten Ländern einbezogen werden, denn durch die jeweilige Stellung innerhalb der Weltarbeitsteilung besteht für alle diese unterent- 
wickelten Länder -- wenn auch in unterschiedlichem Ausmaß - zunächst nur ein geringer Spielraum, sich aus dem Weltmarkt herauszulösen, Gerade durch den Reproduktionszusammenhang dieser Länder mit den Industrienationen müssen solche Versuche notwendigerweise zunächst auf objektive Grenzen stoßen, bevor jene politische Anstrengung zur ökonomischen Realität werden kann.

Zuletzt sei noch auf die 14tägig erscheinenden Chile-Nachrichten hingewiesen, die vom Komitee "Solidarität mit Chile" herausgegeben werden (Kontaktadressen: Clarita Müller-Plantenberg, I Berlin 45, Lorenzstr. 65. Bei Bestellung 10 DM beilegen.) Die Chile-Nachrichten bringen Nachrichten und Analysen und informieren über lokale Komitees, Arbeitsgruppen usw. Das Komitee ,Solidarität mit Chile" sammelt Spenden für die Verfolgten und in Widerstand stehenden Gruppen und hat über zuverlässige Kanäle bereits erhebliche Summen weitergeleitet.

Spendenkonto: Komitee ,Solidarität mit Chile"

PSchKonto Berlin (West) Nr. $380087-108$, Elfriede Kohut,

Abschließend veröffentlichen wir den Text eines Solidaritätsaufrufs, den zwölf am 29./30. 9. 1973 in Frankfurt zusammengetroffene Chile-Komitees als Plattform für ihre weitere A rbeit angenommen haben:

\section{Aufruf zur Solidaritätskampagne für Chile}

Der Griff des chilenischen Militärs nach der Macht war offensichtlich das letzte Mittel der verschiedenen chilenischen und ausländischen Kapitalistengruppen, soziale Veränderungen im Interesse der Mehrheit der Bevölkerung, wie sei von der Regierung der Volkseinheit angestrebt wurden, zu verhindern. Der Militärputsch richtete sich nicht allein gegen eine demokratisch gewählte Regierung, sondern vor allem auch gegen die Organisationen der chilenischen Arbeiter und Bauern, die bereit sind, für ihre Errungenschaften, ihre Fabriken, ihr Land und nicht zuletzt für ihr Leben zu kämpfen.

Die kapitalkräftigen und bevorrechtigten Klassen Chiles hatten bereits vor dem Putsch mit allen Mitteln ihrer wirtschaftlichen Macht, aber auch mit Terror und bürgerkriegsartigen Aktionen versucht, Chaos im Land herzustellen. Nun stellen dieselben gesellschaftlichen Kräfte den Gewaltstreich des chilenischen Militärs als Rettung aus dem Chaos dar, das sie selbst anzurichten bemüht waren.

Dieses Vorhaben zeigt beispielhaft: Das Kapital läßt die Regeln der bürgerlichen Demokratie immer dann bedenkenlos fallen, wenn sich diese gegen Kapitalinteressen auszuwirken drohen.

Der Putsch erfolgte in Kooperation mit imperialistischen Staaten, an ihrer Spitze die USA, die bereits vorher keine Mittel - von der Einmischung der ITT, über Kreditschikanen bis zum Kupferboykott - gescheut hatten, um die Regierung der Volkseinheit zu stürzen. Hinter der Fassade von Entspannungspolitik sind die USA bemüht, soziale Befreiungsbewegungen in Lateinamerika zu liquidieren. 
Wir müssen gegenüber dem bürgerlichen Gewaltstreich in Chile jetzt international dieselbe Solidarität entwickeln, mit der in den vergangenen Jahren die Befreiungsbewegung in Vietnam unterstützt wurde. Wir müssen bewußt machen: gegenüber dem Bruch bürgerlicher demokratischer Legalität in Chile durch Kapital und Militär ist jedes Mittel proletarischer Gegengewalt legitim. Die Errungenschaften der Arbeiter können nur die Arbeiter selbst verteidigen und weiterentwickeln!

Die in Frankfurt versammelten Chile-Komitees aus 12 Städten der Bundesrepublik und Westberlin einigen sich darauf, ihre Bemühungen zu koordinieren, um vor allem folgende Aufgaben zu erfüllen:

1. Informationen zu verbreiten: über den Kampf des chilenischen Volkes, seine Organisationen und seine Errungenschaften während der letzten Jahre, über den Kampf des chilenischen Volkes und seiner Organisationen gegen die faschistische Militariunta, gegen Bourgeoisie und Imperialismus;

2. politische und materielle Aktionen der Solidarität für diesen Kampf und für die Opfer der Unterdrückung in Chile, die sich auch gegen die Exillateinamerikaner in Chile richtet, zu organisieren und zu unterstützen;

3. Druck auf die Bundesregierung, auf nationale und internationale Organisationen auszuüben, um jede Zusammenarbeit mit der Militärjunta zu verhindern und die Unterstützung des chilenischen Widerstandes zu erreichen.

Die in Frankfurt versammelten Vertreter der Chile-Komitees empfahlen den Komitees:

1. die Solidaritätsarbeit für den chilenischen Widerstand so zu gestalten, daß unterschiedliche parteipolitische Interessen die Solidaritätsarbeit nicht behindern;

2. die Mitarbeit in den Komitees allen zu ermöglichen, die mit den Zielen dieses Aufrufes übereinstimmen;

3. eine enge Zusammenarbeit mit den Organisationen der lateinamerikanischen Studenten in der Bundesrepublik und Westberlin anzustreben.

Solidarität für den Sieg der chilenischen Arbeiterklasse! Der Kampf geht weiter!

Frankfurt, 30. 9. 1973

Chile-Solidaritätskomitee Aachen, Lateinamerikanische Gruppe Aachen, Chile-Solidaritätskomitee Düsseldorf, Chile-Aktionskomitee Mainz, AELA Hamburg, ALA Freiburg, ADW Freiburg, Komitee „Solidarität mit Chile" Berlin, KELA Frankfurt, AELA Frankfurt, CLA Heidelberg, Komitee „Solidarität mit Chile“ Heidelberg, Chile-Komitee Tübingen, Chile-Komitee Stuttgart, AELA München. 